\title{
Myomectomy for a Large Submucosal Fibroid at the Time of Caesarean Section
}

\author{
Jian $\mathrm{An}^{1}$, Jinxiao $\mathrm{Lin}^{2}$ and Pengming Sun ${ }^{1}$ \\ ${ }^{1}$ Department of Laboratory of Gynecologic Oncology, Fujian Provincial Maternity and Children's Hospital, Affiliated Hospital of Fujian \\ Medical University, Fuzhou, Fujian, China \\ ${ }^{2}$ Department of Obstetrics, Fujian Provincial Maternity and Children's Hospital, Affiliated Hospital of Fujian Medical University, Fuzhou,
} Fujian, China

\begin{abstract}
Submucosal uterine fibroids are usually associated with infertility and miscarriage. Hence, term pregnancies with large submucosal fibroids are rare in clinical practice. The management of fibroids during pregnancy remains controversial. We, herein, describe a case of natural conception, complicated with a massive submucosal fibroid in a 29-year primigravida. The pregnancy was uneventful with satisfactory fetal growth on serial ultrasound scans until the 4lst week of gestation. At the time of caesarean section, we performed myomectomy successfully after bilateral uterine artery ligation. There was no blood transfused perioperatively. The patient achieved a satisfactory recovery and was discharged on day-4 after surgery. This case indicates that myomectomy during caesarean section may be a safe and feasible intervention in carefully selected cases of submucosal fibroids.
\end{abstract}

Key Words: Myomectomy, Submucosal fibroid, Pregnancy, Caesarean section.

How to cite this article: An J, Lin J, Sun P. Myomectomy for a Large Submucosal Fibroid at the Time of Caesarean Section. J Coll Physicians Surg Pak 2022; 32(03):383-385.

\section{INTRODUCTION}

Uterine fibroids are highly prevalent and have a lifetime incidence of approximately $70 \%$ in the general population. ${ }^{1}$ Currently, fibroids in pregnancy are more commonly encountered in clinical practice than before, in association with the delay in childbearing, and as the use of ultrasound in pregnancy has become more widespread. Prospective studies indicated that the prevalence of fibroids in pregnancy is $10.7 \%$ and that submucosal fibroids account for $17 \%$ of the total. ${ }^{2}$ However, there is a lack of knowledge regarding the management of fibroids during pregnancy and labour. Myomectomy during caesarean section is disputed among obstetricians, as the procedure may increase the risk of uncontrollable haemorrhage and other unexpected complications; however, some studies have indicated that the surgical procedure could be safe and feasible, based on the opinions of the experienced obstetricians. ${ }^{3,4}$ Here, we report a rare and successful case of myomectomy for a large submucosal fibroid at the time of caesarean section.

Correspondence to: Dr. Pengming Sun, Laboratory of Gynecologic Oncology, Fujian Provincial Maternity and Children's Hospital, Affiliated Hospital of Fujian Medical University, Fuzhou, Fujian 350001, China.

E-mail: sunfemy@hotmail.com

Received: December 30, 2019; Revised: February 29, 2020;

Accepted: March 11, 2020

DOI: https://doi.org/10.29271/jcpsp.2022.03.383

\section{CASE REPORT}

We present the case of a 29-year primigravida, who had a history of mild menorrhagia; but she ignored it and had not consulted a doctor. She reported that the pregnancy was spontaneous. She did not know that she had a uterine fibroid until the 6 th week of pregnancy, when an ultrasound scan demonstrated a viable intrauterine pregnancy with a fibroid measuring $10.0 \times 5.5 \times 6.9 \mathrm{~cm}$, located in the right posterior wall of the uterus. Her pregnancy was not closely followed; but seemed uneventful with satisfactory fetal growth on serial ultrasound scans until the 41st week of gestation, when she was admitted to our hospital. The ultrasound showed that the fibroid had increased in size (41st week of gestation: $12.6 \times 8.3 \times 12.3 \mathrm{~cm}$ ) (Figure $1 a, 1 b)$. Vaginal delivery was risky because the intracavitary fibroid may have affected the contractility of the uterus, prolonging the labour process and potentially leading to failure; hence, after careful evaluation, the decision was made to perform a caesarean section.

Before surgery, adequate blood was made available for transfusion, and a vascular surgeon was readily available. During the caesarean section, which was performed under combined spinal epidural anaesthesia, a lower transverse incision was made in the uterus, and a live male baby weighing 3,930 grams was delivered smoothly in good condition. Oxytocin, carbetocin and carboprost were used in our case. However, the surgeon had to manually separate the placenta from the wall of the uterus because there were no signs of natural placental separa- 
tion after a relatively long time. Subsequently, with the contraction of the uterus, a large submucosal fibroid (FIGO class 1 ) was extruded from the uterine cavity through the incision (Figure 2a). Then, myomectomy was performed after bilateral uterine artery ligation to reduce hemorrhage. Suturing the wounds in the upper posterior wall of the uterine cavity was extremely difficult because the fundus of the fibroid was relatively wide, and it was challenging to expose the surgical field. Hence, we inserted compression sutures in the fundus and posterior wall of the uterus, using healthy myometrium as anchoring points for needle transfixion. The stitches penetrated the full thickness of the myometrium; the needle was transfixed from the uterine serosa lateral to the uterine cavity, was threaded along a horizontal course inside the uterine cavity to encompass the wound area, and finally emerged at the uterine serosa lateral. A flat surgical knot was then tied as tightly as possible above the serosa to achieve a better compression effect (Figure 2b, 2c, $2 d)$. No blood was transfused perioperatively. The patient achieved a satisfactory recovery and was discharged on day-4 after surgery. The histological examination revealed submucous leiomyoma measuring $14 \times 12 \times 10 \mathrm{~cm}$ with red degeneration but no malignancy (Figure 2e).
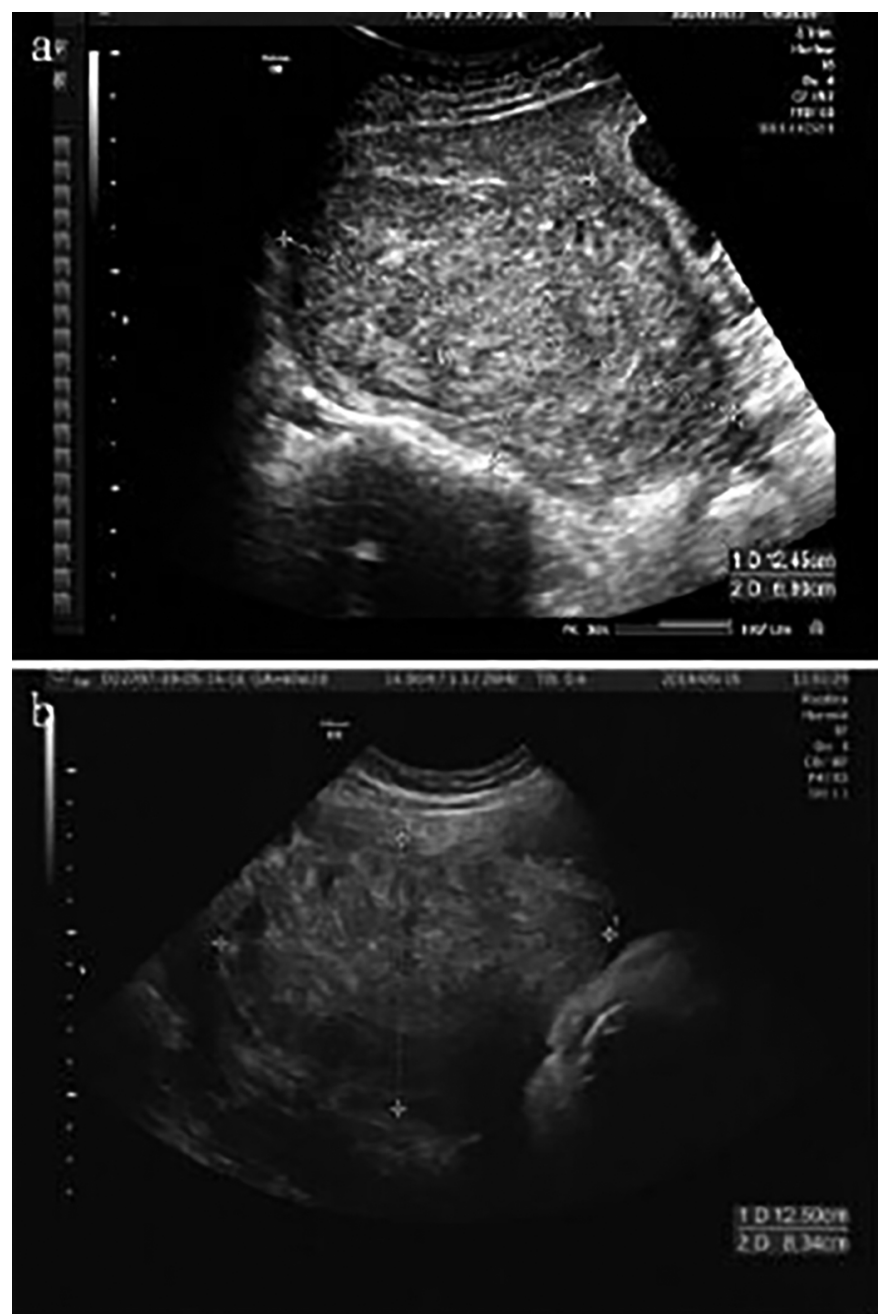

Figure 1: (a) The ultrasound scan on 10thweek of gestation. (b) The ultrasound scan on $39^{\text {th }}$ week of gestation.

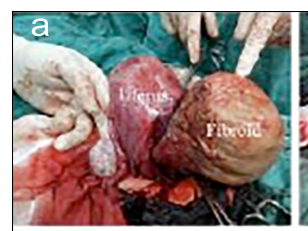

d

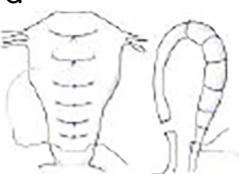

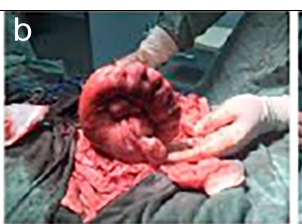

e

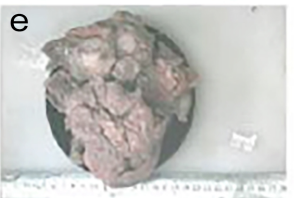

Figure 2: (a) Uterine fibroid exteriorised just before removal from the uterus. (b) Sagittal view of the uterus after suture. (c) Posterior view of the uterus after suture. (d) Schematic illustration of the compression suture. (e) Resected fibroid.

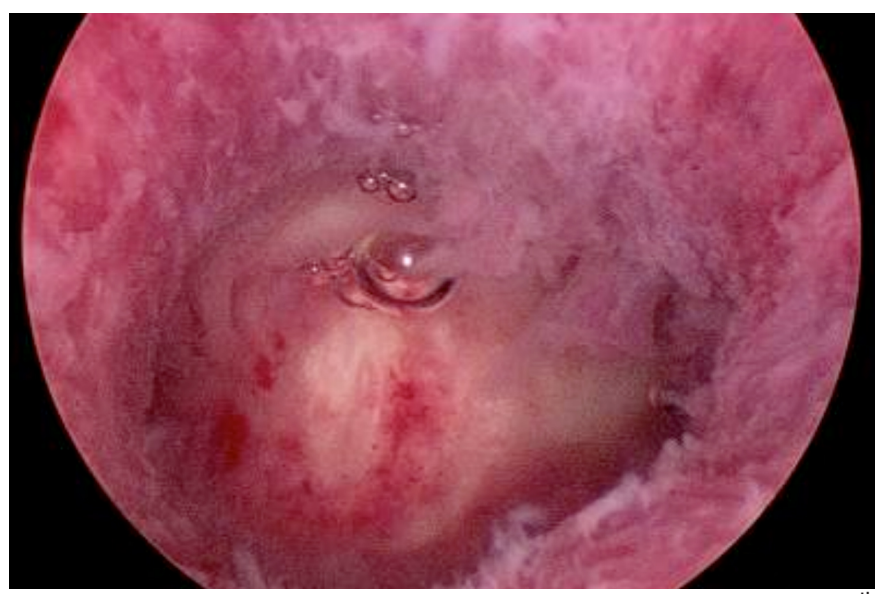

Figure 3: A normal uterine contour is observed on hysteroscopy at $9^{\text {th }}$ month after the caesarean myomectomy.

Ultrasound was performed at the postpartum clinic 42 days after labour, which revealed no apparent retention of the placenta or haematometra. The patient resumed menstruation five months after labour, and there was no distinct alteration in menstruation. A hysteroscopy examination of the uterine cavity was performed nine months after labour, and no intrauterine adhesion was found (Figure 3).

\section{DISCUSSION}

The locations of fibroids can be subserosal, intramural, or submucosal. Subserous and intramural myomas are the most common, while submucosal myomas are rarer. ${ }^{5}$ Large submucosal uterine fibroids are usually associated with infertility, mainly due to the obstructed embryo implantation as a result of uterine cavity morphological changes caused by the space occupied by uterine fibroids. ${ }^{6}$ Even if patients can successfully conceive, fibroids may cause miscarriage, placental abruption, intrauterine growth retardation, intrauterine fetal demise, fetal compression syndrome, preterm birth, and placenta previa. ${ }^{7}$ Thus, our case is quite rare because the pregnancy was spontaneous and uneventful with satisfactory foetal growth.

The management of fibroids in pregnancy remains controversial. Conservative management is common in clinical practice. However, some reports have suggested that myomec- 
tomy can be performed safely in the first and second trimesters of pregnancy; but only in a very carefully selected group of patients. ${ }^{8,9}$ In the present case, myomectomy during pregnancy, seemed unfeasible on account of the submucosal location of the fibroid. On the other hand, the decision to perform myomectomy at the time of caesarean section is also debatable because of the risk of perioperative complications, mainly haemorrhage. ${ }^{10,11}$ A number of recent studies have demonstrated that caesarean myomectomy is a safe and feasible procedure in experienced hands; during the procedure, a large leiomyoma $\geq 5 \mathrm{~cm}$, birth weight $\geq 4,000 \mathrm{~g}$, and a maternal age of $\geq 40$ years may serve as risk factors that are associated with increased blood loss. ${ }^{12}$ For our patient, we decided to perform the myomectomy even though the fibroid size was over $5 \mathrm{~cm}$ because it was difficult to suture the incision in the uterus with the fibroid preserved, and if we forcibly sutured the uterus without removing the fibroid, the fibroid may have hindered the contraction of the uterus due to the space occupied by the fibroid, leading to postpartum haemorrhage. The difficulty faced during surgery is the control of intraoperative haemorrhage. After delivery of the fetus and placenta, we proactively performed bilateral uterine artery ligation to reduce haemorrhage. Furthermore, we creatively sutured the wound from the serosa lateral, which overcame the difficulty in exposing the surgical field and effectively reducing bleeding.

The surgery was very successful and the patient recovered quickly. The prognosis of the patient in terms of menstruation and condition of the uterine cavity was favourable. The patient's next pregnancy needs to be followed so that valuable information can be obtained for use in clinical practice.

In conclusion, we described an unusual case of myomectomy for a large submucosal fibroid at the time of caesarean section. Despite the difficulties encountered in the management of the case during surgery, our management was effective, and the outcome was favourable. However, on the basis of only one case, we cannot conclude that myomectomy at the time of caesarean section is safe. Large randomised trials are needed to justify this conclusion.

\section{PATIENT'S CONSENT:}

Informed consent was taken from the patient to publish his case as a case report.

\section{CONFLICTOF INTEREST:}

The authors declared no conflict of interest.

\section{AUTHORS' CONTRIBUTION:}

JA: Wrote the manuscript and collected images and case details.
JL: Performed the surgery.

PS: Supervised the whole project.

\section{REFERENCES}

1. Marsh EE, Ekpo GE, Cardozo ER, Brocks M, Dune T, Cohen LS. Racial differences in fibroid prevalence and ultrasound findings in asymptomatic young women (18-30 years old): A pilot study. Fertil Steril 2013; 99(7):1951e7. doi: 10.1016/j. fertnstert.2013.02.017.

2. Laughlin SK, Baird DD, Savitz DA, Herring AH, Hartmann $\mathrm{KE}$. Prevalence of uterine leiomyomas in the first trimester of pregnancy: An ultrasoundscreening study. Obstet Gynecol 2009; 113(3):630-5. doi: 10.1097/AOG.0b013e318 197 bbaf.

3. Zhao R, Wang X, Zou L, Zhang W. Outcomes of myomectomy at the time of cesarean section among pregnant women with uterine fibroids: A retrospective cohort study. Biomed Res Int 2019; 2019:7576934. doi: 10.1155/2019/7576934.

4. Pergialiotis V, Sinanidis I, Louloudis IE, Vichos T, Perrea DN, Doumouchtsis SK. Perioperative complications of cesarean delivery myomectomy: A meta-analysis. Obstet Gynecol 2017; 130(6):1295. doi: 10.1097/AOG.0000000000002342.

5. Buttram VC Jr, Reiter RC. Uterine leiomyomata: Etiology, symptomatology and management. Fertil Steril 1981; 36(4):433-45. doi: 10.1016/s0015-0282(16)45789-4.

6. Somigliana S, Vercellini P, Daguati R, Pasin R, De Giorgi O, Crosignani PG. Fibroids and female reproduction: A critical analysis of the evidence. Hum Reprod Update 2007; 13(5): 465-76. doi: 10.1093/humupd/dmm013.

7. Milazzo GN, Catalano A, Badia V, Mallozzi M, Caserta D. Myoma and myomectomy: Poor evidence concern in pregnancy. J Obstet Gynaecol Res 2017; 43(12):1789-804. doi: $10.1111 /$ jog.13437.

8. Lolis DE. Successful myomectomy during pregnancy. Hum Reprod 2003; 18(8):1699-702. doi: 10.1093/humrep/ deg318.

9. Celik C, Acar A, Cicek N, Gezginc K, Akyürek C. Can myomectomy be performed during pregnancy? Gynecol Obstet Invest 2002; 53(2):79-83. doi: 10.1159/000052997.

10. Song D, Zhang W, Chames MC, Guo J. Myomectomy during cesarean delivery. Int J Gynaecol Obstet 2013; 121(3): 208-13. doi: 10.1016/j.ijgo.2013.01.021.

11. Sparic R, Guido M, Tinelli A. Cesarean myomectomy and possible risk factors for admission to intensive care unit - a retrospective study. Ginekol Pol 2015; 86(10):731-6. doi: $10.17772 / \mathrm{gp} / 57828$.

12. Dedes I, Schäffer L, Zimmermann R, Burkhardt T, Haslinger $C$. Outcome and risk factors of cesarean delivery with and without cesarean myomectomy in women with uterine myomatas. Arch Gynecol Obstet 2017; 295(1):27-32. doi: 10.1007/s00404-016-4177-8. 